\title{
New results on GP Com
}

\author{
L. Morales-Rueda ${ }^{1}$, T. R. Marsh ${ }^{1}$, D. Steeghs ${ }^{1,2}$, E. Unda-Sanzana ${ }^{1}$, J. H. Wood ${ }^{3}$, and R. C. North ${ }^{1,4}$ \\ 1 Department of Physics and Astronomy, Southampton University, Southampton SO17 1BJ, UK \\ e-mail: 1mr@astro.soton.ac.uk; trm@astro.soton.ac.uk \\ 2 Harvard-Smithsonian Center for Astrophysics, 60 Garden Street, Cambridge, MA 02318, USA \\ e-mail: dsteeghs@head-cfa.harvard.edu \\ 3 Astrophysics Group, School of Chemistry and Physics, Keele University, Staffordshire, ST5 5GB, UK \\ ${ }^{4}$ Met Office, London Road, Bracknell, Berkshire, RG12 2SZ, UK
}

Received 14 January 2003 / Accepted 11 April 2003

\begin{abstract}
We present high resolution optical and UV spectra of the $46 \mathrm{~min}$ orbital period, helium binary, GP Com. Our data contains simultaneous photometric correction which confirms the flaring behaviour observed in previous optical and UV data. In this system all lines show a triple peaked structure where the outer two peaks are associated with the accretion disc around the compact object. The main aim of this paper is to constrain the origin of the central peak, also called "central spike". We find that the central spike contributes to the flare spectra indicating that its origin is probably the compact object. We also detect that the central spike moves with orbital phase following an $\mathrm{S}$-wave pattern. The radial velocity semiamplitude of the $\mathrm{S}$-wave is $\sim 10 \mathrm{~km} \mathrm{~s}^{-1}$ indicating that its origin is near the centre of mass of the system, which in this case lies very close to the white dwarf. Our resolution is higher than that of previous data which allows us to resolve structure in the central peak of the line. The central spike in three of the He I lines shows another peak blueshifted with respect to the main peak. We propose that one of the peaks is a neutral helium forbidden transition excited in a high electron density region. This forbidden transition is associated with the permitted one (the stronger peak in two of the lines). The presence of a high electron density region again favours the white dwarf as their origin. We compute Doppler maps for the emission lines which show three emission regions: an accretion disc, a bright spot and an unidentified low velocity emission region associated with the central spike. We obtain modulation Doppler tomograms for some of the emission lines that map the anisotropic emission from the bright spot region. The HST UV spectra also show a strong flare component and confirm the under abundance of silicon in GP Com.
\end{abstract}

Key words. accretion, accretion discs - binaries: spectroscopic - line: profiles - stars: mass-loss stars: novae, cataclysmic variables - stars: individual: GP Com.

\section{Introduction}

The AM CVn stars are close binaries in which a white dwarf accretes from the stripped-down core of a giant star or a helium white dwarf (Tutukov \& Federova 1989). Three possible channels have been discussed in the literature regarding the formation of AM CVn systems: (a) a double degenerate channel (Tutukov \& Yungelson 1979), (b) a channel in which the donor is a semi-degenerate star (Savonije et al. 1986) and (c) a channel in which the donor starts as a non-degenerate post main sequence star (Podsiadlowski et al. in preparation). Near relatives of the cataclysmic variables (CVs), the AM CVn stars have periods ranging from 5 to $65 \mathrm{~min}$, and accretion discs composed of $>99$ per cent helium by number, giving us a unique opportunity to test the influence of composition upon disc physics. Although only 8 or 9 of these systems are known, they are predicted in some studies to have a space density about a factor of 2 higher than that of the CVs of which some 700 are known (Tutukov \& Yungelson 1996). Other studies predict an observable population that agrees with the observed one
(Nelemans et al. 2001). AM CVn systems may be significant sites of accretion-induced collapse producing either neutron stars or type Ia supernovae, a possibility denied to the CVs because of erosion of their white dwarfs in nova explosions (Tutukov \& Yungelson 1996).

The theory of binary star evolution predicts that most AM CVn systems have low accretion rates (Tutukov \& Yungelson 1996). The disc instability model (Osaki 1974) predicts that at low accretion rates, discs can exist in a steadystate. Moreover, the threshold below which the discs are steady is higher for helium than hydrogen discs, a consequence of the higher ionisation energy of helium (Tsugawa \& Osaki 1997). As a result most AM CVn systems should be faint, and may fail to outburst, making them hard to spot. Of the known systems, GP Com (=G61-29), which has an orbital period of $46 \mathrm{~min}$, is intrinsically faint $\left(M_{V}>11\right)$ and has never shown outbursts, is closest to the properties expected of the bulk of AM CVns (Tutukov \& Yungelson 1996). GP Com's components are thought to be a $\sim 0.5 M_{\odot} \mathrm{CO}$ white dwarf accreting from a $0.02 M_{\odot}$ helium degenerate (Nather et al. 1981). 
GP Com's optical spectrum, which is full of He I and He II lines, resembles very closely that of a recently discovered AM CVn system, CE315 (Ruiz et al. 2001); the orbital period of CE315 is $65 \mathrm{~min}$. In the UV wavelength range, GP Com shows a plethora of nitrogen lines and possibly some C I but no sign of the CIV or Si IV commonly seen in CVs. Lambert \& Slovak (1981) suggest that this is the result of the CNO process depleting the carbon and enhancing the nitrogen. Marsh et al. (1991) model the optical lines with an LTE model and obtain a reasonable fit, which confirms the CNO processing, but with very peculiar abundances of heavy elements such as silicon, calcium and iron which appear to be under abundant compared to the Sun by 100 to 1000 times.

GP Com flares erratically at X-ray (van Teeseling \& Verbunt 1994), UV (Marsh et al. 1995) and optical wavelengths (Marsh et al. 1991). The variability is strongest in high ionisation lines, implicating the inner disc, as do the shorttimescales involved. This suggests that the flaring is driven by irradiation of the disc by a variable source at the centre of the disc. The flaring causes large changes in the line profiles (Marsh et al. 1995). The line profiles show evidence for being ionisation-bounded (Marsh 1999).

In this paper we study the source of the flaring and in particular the contribution of the mystery spike at the centre of each line to the line flaring. The origin of the central spike has been suggested to be a nebula (Nather et al. 1981), but none has been found (Stover 1983). Instead we see that the spike may participate in flaring (Marsh 1999), which unlikely though it seems, leaves the accreting white dwarf as its most plausible origin. A potential problem of Marsh's (1999) study was that the spectra were only approximately photometric, with corrections based upon a comparison star on the slit. This could have made the central spike appear to be varying artificially.

We obtain modulation Doppler tomograms that map the flux variability seen in the bright-spot region. We also present HST UV data and study the flaring component in the UV spectra.

\section{Observations}

We obtained spectroscopy of GP Com on the nights of 1999 March 26 and 27, using the ISIS double beam spectrograph mounted on the $4.2 \mathrm{~m}$ William Herschel Telescope (WHT) on La Palma. Table 1 gives a journal of observations.

For the red arm, the standard readout mode was used in conjunction with a Tektronix CCD $(1 \mathrm{k} \times 1 \mathrm{k})$ windowed to $1124 \times 650$ pixels to reduce dead time. Exposure times were generally $180 \mathrm{~s}$, for the first night and $300 \mathrm{~s}$ during the second night (when the seeing got worse) with $\sim 33 \mathrm{~s}$ dead time, and the spectral resolution corresponds to $56 \mathrm{~km} \mathrm{~s}^{-1}$ at $\lambda 7067 \AA$. The 600 lines $\mathrm{mm}^{-1}$ grating R600R was used to cover the wavelength range $\lambda \lambda 6600-7408 \AA$ during the first night and $\lambda \lambda 7972-8784 \AA$ during the second. A total of 81 and 45 spectra were obtained during the first and second nights respectively.

For the blue arm, the standard readout mode was used on the EEV12 CCD $(4 \mathrm{k} \times 2 \mathrm{k})$ windowed to $1100 \times 4200$ pixels. Exposure times were $180 \mathrm{~s}$ the first night and $300 \mathrm{~s}$ the second with $47 \mathrm{~s}$ dead time. The spectral resolution corresponds
Table 1. Journal of optical observations. $E$ is the cycle number plus binary phase with respect to the ephemeris given by Marsh (1999).

\begin{tabular}{cccccc}
\hline \hline Date & $\begin{array}{c}\text { Start } \\
(\mathrm{UT})\end{array}$ & $\begin{array}{c}\text { End } \\
\text { WHT/Red }\end{array}$ & & $\begin{array}{c}\text { Start } \\
(E-123000)\end{array}$ & $\begin{array}{c}\text { End } \\
\text { frames }\end{array}$ \\
\hline 26/3/99 & 23.12 & 5.55 & 374.814 & 383.102 & 81 \\
27/3/99 & 22.86 & 5.82 & 405.403 & 414.370 & 45 \\
WHT/Blue & & & & & \\
26/3/99 & 23.12 & 5.57 & 374.815 & 383.121 & 81 \\
27/3/99 & 22.86 & 5.83 & 405.402 & 414.379 & 51 \\
JKT & & & & & \\
26/3/99 & 23.72 & 5.62 & 375.577 & 383.185 & 232 \\
$27 / 3 / 99$ & 22.17 & 4.88 & 404.509 & 413.155 & 224 \\
HST & & \multicolumn{5}{c}{$(E-76000)$} & \\
$4 / 2 / 95$ & 17.96 & 20.99 & 643.752 & 647.654 & 13 \\
\hline
\end{tabular}

to $38 \mathrm{~km} \mathrm{~s}^{-1}$ at $\lambda 4658 \AA$. The 1200 lines $\mathrm{mm}^{-1}$ grating R1200B was used to cover the wavelength range $\lambda \lambda 4253-5058 \AA$ enabling simultaneous monitoring of several He I lines and He II $\lambda 4686 \AA$. A total of 132 spectra were obtained during both nights.

After debiasing and flat-fielding the frames by tungsten lamp exposures, spectral extraction proceeded according to the optimal algorithm of Marsh (1989). The data were wavelength calibrated using $\mathrm{CuAr}$ and $\mathrm{CuNe}$ arc lamps. The arcs were extracted using the profile associated with their corresponding target to avoid systematic errors caused by the spectra being tilted. The spectra were then corrected for instrumental response and extinction using the flux standard BD332642 (Stone 1977). The telluric standard HR5718 (Osawa 1959) was used to correct for the atmospheric water absorption bands. Uncertainties on every point were propagated through every stage of the data reduction.

Simultaneous $B$-band photometry of GP Com was taken during both nights using a Harris $B$ filter with a Tektronix CCD $(1 \mathrm{k} \times 1 \mathrm{k})$ on the $1.0 \mathrm{~m}$ Jacobus Kapteyn Telescope (JKT). Exposure times during the first night ranged between 10 and $40 \mathrm{~s}$ depending on the seeing. The seeing worsened during the second night thus the exposure times had to be increased to values between 50 and $75 \mathrm{~s}$. These data were used to calibrate the blue spectra in flux. Therefore any variations present in the blue spectra after this calibrations would be intrinsic and not due to slit losses or atmospheric variations. We obtained a total of 118 calibrated spectra after these corrections.

We also present 13 UV spectra of GP Com taken with HST on the 4th of February 1995 using the G140L grating on the High Resolution Spectrograph (HRS). The wavelength range covered by the spectra is $\lambda \lambda 1150 \AA-1440 \AA$ with a dispersion of $0.572 \AA$ per diode. Exposure times were of the order of $400 \mathrm{~s}$. The data were reduced using the pipeline software. Details of the observing times and orbits covered are given in Table 1. 

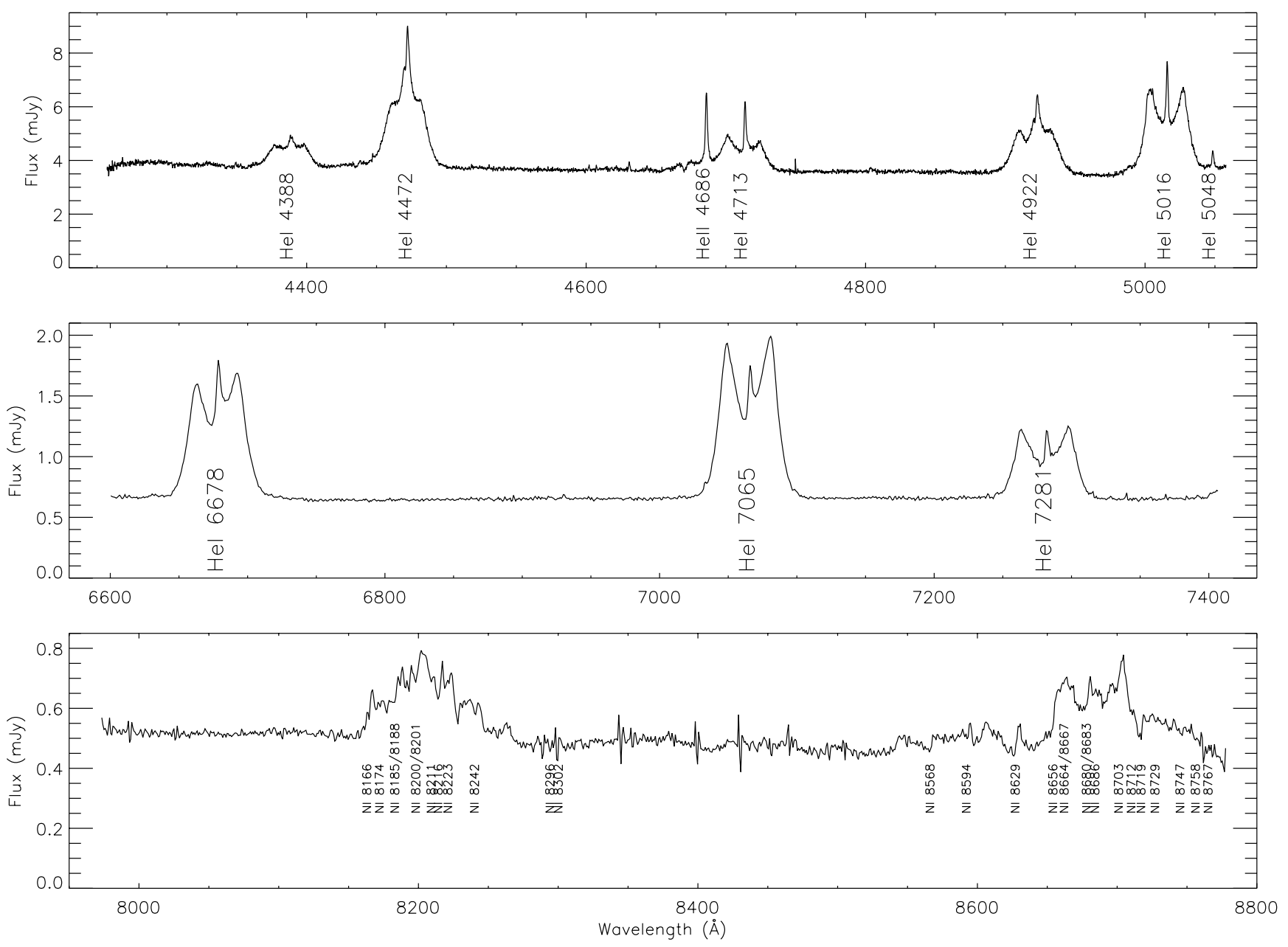

Fig. 1. Average spectra of all our GP Com data for the three wavelength ranges observed. The most prominent lines are labelled.

\section{Results}

\subsection{Average spectra}

The profiles in GP Com consist of a classic double-peaked line from the disc plus a narrow "spike" at the centre of each line (see Fig. 1). The double-peaked lines are formed by Doppler shifting with the line wings coming from close to the white dwarf while the peaks themselves come from the outer edge of the emission region. Figure 1 presents the average spectra obtained for each wavelength range observed during this campaign. The top spectrum can be directly compared with Fig. 1 of Marsh (1999) and Fig. 1 of Nather et al. (1981). Although Nather et al.'s spectra were not flux calibrated we can still compare the relative strength of the central spike with respect to the wings of the line between the three different sets of data. In Marsh's and Nather et al.'s spectra the strength of the central spike relative to the wings of the lines is comparable. In our spectrum the wings of the lines are as strong as those presented by previous authors but the central spike is $\sim 1.7$ times stronger indicating that GP Com shows long term variability and evidence that the spike participates in the variability. In Marsh (1999) the signal to noise ratio of the data was not high enough to confirm that the central spike was varying. The central spike is clearly red-shifted in all emission lines apart from $\mathrm{He} \mathrm{I} \lambda 5016 \AA$, the red-shift being different for each line (see Table 3) as already noticed by Marsh (1999).

A new feature present in our data is the absorption located to the blue of He II $\lambda 4686 \AA$. The fact that this absorption was not present in Marsh's (1999) spectra indicates that it is a transient feature.

The resolution of the spectrum in the middle panel (wavelength range $\lambda \lambda 6600-7408 \AA$ ) allows us to see the central spike (red-shifted also in these cases) for three more He I lines. The spectrum in the lower panel shows a complicated combination of many $\mathrm{N}$ I lines, but none of any other element.

\subsection{Flares}

GP Com displays strong flaring in X-ray (van Teeseling \& Verbunt 1994), UV (Marsh et al. 1995) and optical (Marsh et al. 1991) wavelengths. This variability is clearly present in our data, mainly in the emission lines. Figure 2 shows the light curves of the continuum and blue emission lines over this two day run. The blue data $(\lambda \lambda 4253-5058 \AA)$ presented in this work are photometric, as we used simultaneous photometry to correct from atmospheric variations in the spectra, therefore any variation observed in the data is intrinsic. This is confirmed by 


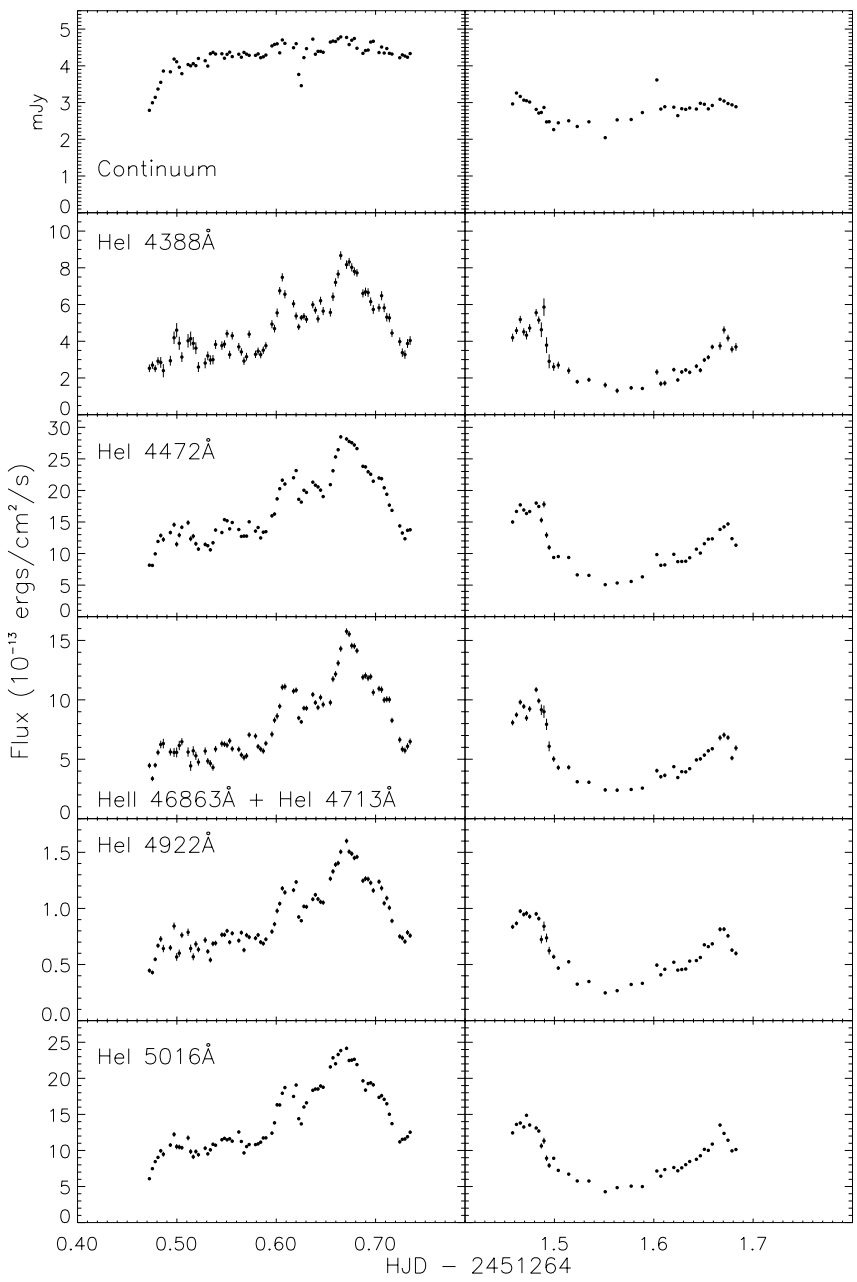

Fig. 2. Light curves of the blue continuum and several emission lines found in the blue spectra. GP Com shows strong variability in all the lines. This variability does not seem to be due to its orbital motion.

the fact that we see the same variations in all the blue lines (Fig. 2).

The lightcurves obtained for both nights are very different. The variability seen in the lines and the continuum is also different, the continuum varying less significantly than the lines. For the emission lines, we see that during the first night GP Com shows short term flares that last of the order of 15 to $70 \mathrm{~min}$. These flares are present throughout the entire night. During the second half of the first night these short timescale flares are superimposed to a longer timescale brightening in the lines that lasts of the order of 3 hours. On the other hand, during the second night, although we also see short timescale flares, these take place mainly at the beginning and at the end of the observations with very little variability in between. We searched for periodicities in the lightcurves of the emission lines by using a floating mean periodogram (Cumming et al. 1999) and found nothing significant. All the emission lines show similar variability.

As for the continuum, the variability observed is smaller than that seen in the emission lines, 10 to 20 per cent during the first night and up to 30 per cent during the second night compared with variations of up to 80 per cent in the emission lines. During the second night the variations seen in the continuum seem to follow those seen in the lines, i.e. a flare at the beginning of the observations followed by a decrease and perhaps a general increase at the end of the night. This is not so clear during the first night, although the initial increase seen in the continuum and the decrease at HJD - $2451264=0.63$ match the behaviour seen in He I $\lambda 5016 \AA$. A search for periodicities in the continuum lightcurves, using the same method as for the emission lines yields no significant period, in particular the orbital period is not present.

We used the same method described in Marsh et al. (1995) to obtain the spectrum for the flaring component. This consists of modelling the spectra as the sum of two components, one of them being the mean spectrum and the other a multiple of the flare component. The model fitting is carried out iteratively until the two spectra and the multipliers are optimised by minimising $\chi^{2}$. All the spectra are used to obtain the mean and the flare components because during the calculations each spectrum is weighted according to its total flux giving the highest signal to noise ratio in the flare spectrum. The average and flare components of the spectra are shown in Fig. 3. The calculation of the flare spectrum was made by using the raw spectra (middle panel) and the continuum normalised spectra (bottom panel). By using the continuum normalised spectra we can investigate whether the broad component of the lines and the central spike vary in the same way as the continuum.

The flare spectra obtained in these two cases are noticeably different, i.e. the central spike does not seem to be present in many of the emission lines in the flare spectrum obtained from the continuum normalised spectra but it is present in all lines for the raw spectra. The broad component of the helium lines is comparable in both cases which indicates that they do not vary in a similar way to the continuum, i.e. broad line and continuum variations seem to be independent. On the other hand the central spike does look different in both flare spectra. For He II $\lambda 4686 \AA$ and He I $\lambda 4713 \AA$ the central spike is $\sim 1.5$ times stronger in the raw spectrum, and for all the other helium lines the central spike is present. This indicates that the central spike component varies in a similar way, but not identical, to the continuum. Where this variability is produced is still an unknown but we can at least say that the variability in the broad lines has a different origin to that in the continuum and the central spike. If we assume that the optical variability is the result of irradiation by the emitted X-rays, the flare spectra suggest that the broad component of the lines is formed in an optically thin region, more responsive to irradiation, whereas the continuum and the central spike have their origin in a region less responsive to irradiation, probably optically thick.

The behaviour seen in the $\mathrm{N}$ I lines is different to that of the helium lines. In this case the lines also contribute to the flare spectrum but their variability is not independent from the continuum variability. Continuum and lines vary in similar ways which is why we see very little signal in the flare spectrum obtained from the continuum normalised spectra.

The main question we wanted to answer in this section was whether the central spike contributed to the flare spectrum as suggested by Marsh (1999). From Fig. 3 we see that this is the case. From this we conclude that the origin of the central 

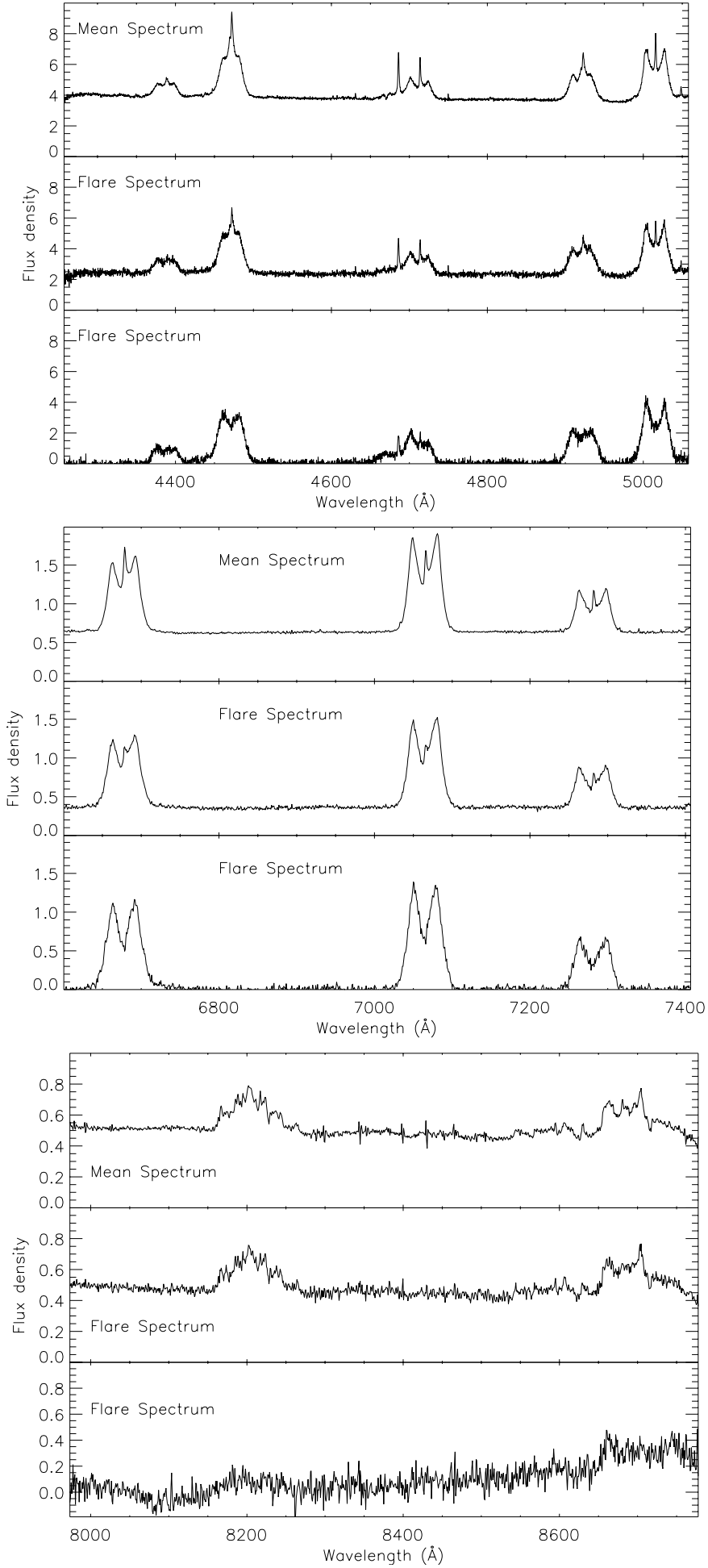

Fig. 3. The panels show the mean GP Com spectrum (top), the flare spectrum obtained from the raw spectra (middle) and the flare spectrum obtained from the continuum normalised spectra (bottom).

spike seems to be the accreting star and not a nebula around the system.

Another important difference found between the average and flare spectra can be seen in Fig. 4 where we have plotted both components for He I $\lambda 6678 \AA$, He I $\lambda 7065 \AA$ and He I $\lambda 7281 \AA$. In all cases the flare component is broader

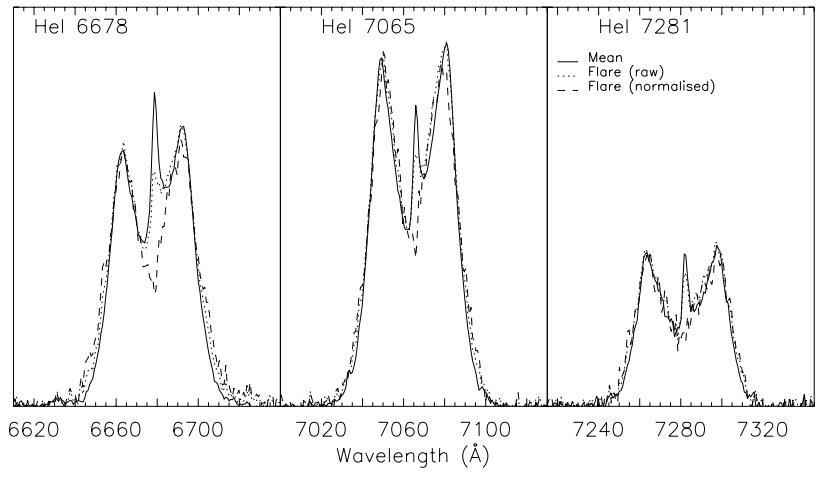

Fig. 4. Average and flare components for the lines He I $\lambda 6678 \AA$, He I $\lambda 7065 \AA$ and He I $\lambda 7281 \AA$. The flare components (dotted and dashed lines) have been scaled to match the average component of the lines. Notice that the flare profiles are broader than the average profiles indicating that they form at higher velocity regions.

Table 2. Changes in FWZI between the helium lines in the mean and flare spectra. The flare spectrum used for these measurements was that obtained from the continuum normalised spectra. Changes in FWZI between the mean and flare spectra obtained from the raw data are about 0.5 times those shown.

\begin{tabular}{lcc}
\hline \hline Line & \multicolumn{2}{c}{$\Delta F W Z I$} \\
& $\mathrm{~km} \mathrm{~s}^{-1}$ & $\AA$ \\
\hline He I 4388 & 399 & 5.8 \\
He I 4472 & 526 & 7.8 \\
He II 4686 + He I 4713 & 353 & 5.5 \\
He I 4922 & 149 & 2.4 \\
He I 5016 & 318 & 5.3 \\
He I 6678 & 455 & 10 \\
He I 7065 & 156 & 3.7 \\
He I 7281 & 84 & 2 \\
\hline
\end{tabular}

than the average component indicating that flaring occurs mainly in high velocity regions, i.e. the inner regions of the disc. Table 2 gives a list of the change in full width at zero intensity $(F W Z I)$ between the mean and the flare spectrum obtained from the continuum normalised data. FWZI changes between the mean spectra and the flare spectra obtained from the raw data are about half those values given in Table 2 .

\subsection{Central spike}

Once we have established that the central spike contributes to the flaring component of the spectra and therefore that its origin is probably the white dwarf, it is of great interest to measure its radial velocity amplitude to further constrain its origin. We carry out this measurement in the same way described by Marsh (1999), by using multi-Gaussian fitting to the profile of the line. We used the raw spectra to carry out the fitting, therefore the mean and flare components are fitted simultaneously. Each emission line is fitted with four Gaussians, one for each wing, one for the bright spot component and one for the central spike. In the case of He II $\lambda 4686 \AA$ only one Gaussian was used to fit the profile as the right wing was heavily contaminated by He I $\lambda 4713 \AA$, the left wing showed strong absorption and the bright spot emission was very faint (see Fig. 5). 


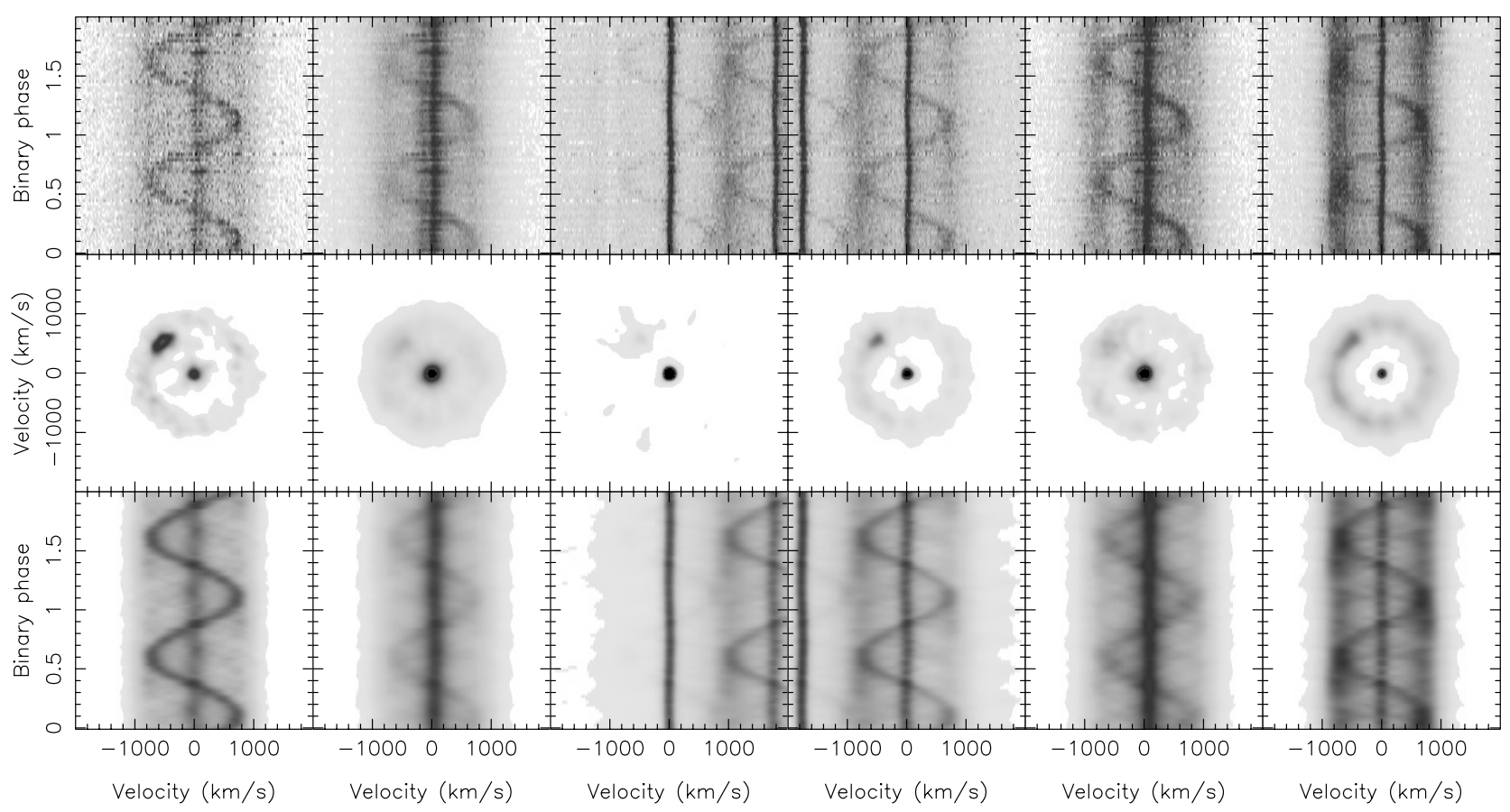

Fig. 5. Trails of the lines (top panels), Doppler maps (middle panels) and trails of the lines computed back from the maps (bottom panels). We have corrected the ephemeris given by Marsh (1999) so the modulation of the central spike corresponds to the motion of the compact object. Emission is clearly seen in the position corresponding to the bright spot.

For He I $\lambda 4472 \AA$ and He I $\lambda 4922 \AA$ two Gaussians instead of one were used to fit the central spike component as this included the satellite feature discussed in the following section. This was not possible for $\mathrm{HeI} \lambda 4388 \AA$ as the line is significantly fainter than He I $\lambda 4472 \AA$ and He I $\lambda 4922 \AA$. The values of the fitting parameters that most interest us are those measured for the central spike component. By fitting all the spectra simultaneously we obtained an optimal value for the width of the spike component. We then maintained this parameter fixed and left two parameters variable, the height of the Gaussian and its offset from the rest wavelength. The parameters for the other Gaussians used were left free. The velocities measured for the central spike (equivalent to the offsets) were then fitted with a sinusoidal function of period equal to GP Com's orbital period, $V=\gamma+K_{\mathrm{cs}} \sin \left(2 \pi\left(\phi-\phi_{0}\right)\right)$, where $\gamma$ is the systemic velocity, $K_{\mathrm{cs}}$ is the radial velocity semiamplitude of the central spike component, $\phi$ is the orbital phase and $\phi_{0}$ is the phase at which the radial velocity semiamplitude is zero. This is equivalent to fitting the data with a function $V=\gamma-V_{X} \cos 2 \pi \phi+V_{Y} \sin 2 \pi \phi$ where $V_{X}$ and $V_{Y}$ are just a combination of the radial velocity semiamplitude and the orbital phase. We present the fitting parameters in Table 3. The problem of the redshift of He I $\lambda 5016 \AA$ being significantly smaller than that of the other lines found by Marsh (1999) remains in our data.

\subsubsection{The origin of the double-peaked central spike}

The lines He I $\lambda 4388 \AA$, He I $\lambda 4472 \AA$ and He I $\lambda 4922 \AA$ share a feature not seen in other lines: the central spike has a
Table 3. Velocity parameters of the central spike for some of the emission lines.

\begin{tabular}{lccc}
\hline \hline Line & $\begin{array}{c}\gamma \\
\mathrm{km} \mathrm{s}^{-1}\end{array}$ & $\begin{array}{c}V_{X} \\
\mathrm{~km} \mathrm{~s}^{-1}\end{array}$ & $\begin{array}{c}V_{Y} \\
\mathrm{~km} \mathrm{~s}^{-1}\end{array}$ \\
\hline He I 4388 & - & - & - \\
He I 4472 & $47.00 \pm 0.80$ & $13.43 \pm 1.12$ & $5.37 \pm 0.58$ \\
He II 4686 & $20.67 \pm 0.29$ & $10.32 \pm 0.42$ & $0.75 \pm 0.09$ \\
He I4713 & $38.34 \pm 0.54$ & $11.82 \pm 0.76$ & $3.43 \pm 0.33$ \\
He I4922 & $64.91 \pm 0.89$ & $10.71 \pm 1.41$ & $0.17 \pm 0.22$ \\
He I5016 & $8.85 \pm 0.53$ & $10.36 \pm 0.73$ & $2.68 \pm 0.30$ \\
He I6678 & $29.04 \pm 0.68$ & $12.37 \pm 1.03$ & $0.38 \pm 0.18$ \\
He I7065 & $36.62 \pm 0.61$ & $11.06 \pm 0.88$ & $0.26 \pm 0.16$ \\
He I7281 & $37.17 \pm 1.00$ & $13.37 \pm 1.42$ & $2.24 \pm 0.46$ \\
\hline
\end{tabular}

satellite blueward of the main peak. In He I $\lambda 4388 \AA$, this satellite is comparable to the main peak whereas for the other two lines the satellite is weaker than the main peak. These can be seen most clearly in the trailed spectra of Fig. 5. We believe these features are the result of Stark effect.

The presence of charged particles in the gas makes Stark broadening an important effect to look for in the spectra of GP Com. In the case of hydrogen, emission and absorption lines split symmetrically and, if the electric field is not very strong, the hydrogen line will appear broadened. In the case of neutral helium, the presence of the charged particles in the plasma causes the upper and lower energy states of a permitted transition to mix with their neighbouring states. This results in forbidden neutral helium transitions being excited (Beauchamp \& Wesemael 1998). A given neutral helium forbidden 
Table 4. Columns 1 and 2 show the wavelengths of the forbidden and permitted helium transitions respectively. The expected separations in wavelength between forbidden and permitted transitions are shown in Col. 3. Column 4 gives a measurement of the expected relative strength of each forbidden transition. These first four columns are taken from Table 1 of Beauchamp \& Wesemael (1998). The last column presents the separations we measure between peaks in our data. $T_{4}$ is the local temperature in units of $10000 \mathrm{~K}$.

\begin{tabular}{lllll}
\hline \hline Forbidden & Permitted & $\lambda_{\mathrm{f}}-\lambda_{\mathrm{p}}(\AA)$ & $\log \left(\chi_{\text {forb }} / \kappa_{\nu}\right)$ & $\Delta \lambda$ \\
\hline 4387 & 4388 & -0.54 & $5.67-0.13 / T_{4}$ & $1.25 \pm 0.28$ \\
4470 & 4472 & -1.48 & 5.18 & $2.57 \pm 0.28$ \\
4921 & 4922 & -1.32 & $4.83-0.13 / T_{4}$ & $2.86 \pm 0.27$ \\
4383 & 4388 & -4.65 & $3.74-0.13 / T_{4}$ & - \\
4911 & 4922 & -11.2 & $3.04-0.13 / T_{4}$ & - \\
4517 & 4472 & +46.0 & 2.26 & - \\
5042 & 5016 & +26.4 & $1.44+0.17 / T_{4}$ & - \\
\hline
\end{tabular}

transition is therefore associated with a permitted one. These forbidden neutral helium lines have been observed in many helium rich white dwarfs (DB white dwarfs; Beauchamp et al. 1995, 1997).

Beauchamp \& Wesemael (1998) in their Table 1 give a list of forbidden transitions that might be found in helium rich stars and a measure of the probability with which we should see them in their spectra. The order in which the forbidden components appear in the list is the order in which they should become observable. We present an extract of their results together with some measurements from our data in Table 4 . The table only presents the predictions for the lines covered by our wavelength range. The first and second columns give the values of the forbidden and permitted transitions respectively. The third column shows the separation in Å expected between both components. The fourth column shows the ratio of the line opacity associated with the forbidden component, $\chi$ forb, to the local continuum opacity, $\kappa_{v}$, as a measure of the relative strength of the forbidden component in the spectrum. If one of the forbidden transitions listed in the table is present in the spectra then all forbidden transitions with values of $\chi_{\text {forb }} / \kappa_{v}$ larger than that of the observed transition should also be present. The last column in Table 4 gives the separation between the two components of the central spike seen our spectra. According to Beauchamp \& Wesemael's (1998) argument, the fact that we see two components in He I $\lambda 4922 \AA$ implies that we should also see them in He I $\lambda 4472 \AA$ and He I $\lambda 4388 \AA$ which agrees with our observations. The predicted separations between the forbidden and permitted transitions do not agree but they seem to follow the same trend, i.e. the separation between both components in He I $\lambda 4388 \AA$ is significantly smaller than for the other two lines, and for these the separations are comparable. In fact, the predicted and observed wavelength separations seem to differ by a factor of $\sim 2$. We do not have an explanation for this discrepancy.

The relevance of this result is enormous because it could cause the lines to be shifted around by different amounts, and also, as there is an asymmetry involved, it could be the explanation for the different redshifts observed for the central spike in different lines.

A direct consequence of this claim is the prediction of the presence in the spectrum of GP Com of the forbidden helium components that have larger values of $\chi_{\text {forb }} / \kappa_{v}$ than the ones observed in our data. These lines, given in
Beauchamp \& Wesemael's (1998) table, are 3705, 4009, 3820, 4144 and $4026 \AA$. It is worth pointing out that the separations expected between the forbidden and permitted components for He I 3705, 4009, 3820 and $4144 \AA$ are smaller than those measured here and higher resolution spectra will probably be required to be able to resolve both components. In the case of the $4026 \AA$ line, the separation expected is larger than of He I $\lambda 4388 \AA$ (which is in the limit of our resolution) so spectra of similar resolution to the ones presented here will suffice to test our prediction.

According to Beauchamp \& Wesemael (1998), the profiles of these neutral helium forbidden components are good indicators of the density and gravity of the medium in which they form. The denser the medium the more symmetrical and wider the profiles. Also the higher the gravity the more symmetric the profiles appear. It would be very interesting to apply model atmosphere codes that have been developed for DB white dwarfs to GP Com and similar systems to calculate the density and gravity of the region where the central spike forms.

This study should be carried out also for the recently discovered twin system of GP Com, CE315. Being so similar to GP Com we might expect to find that the Stark effect is also of importance and that the neutral helium forbidden components are present in its spectrum.

\subsection{Doppler maps}

We present the trails of the helium emission lines present in the spectra in the top panels of Figs. 5 and 6 . The spectra have been rescaled to take away the line flux variations resulting from GP Com's flaring. The ephemeris calculated by Marsh (1999) is not precise enough to be extrapolated thus we used a corrected ephemeris to bin and fold the data:

$\mathrm{HJD}=2451200.2745+0.0323386 \mathrm{E}$.

This corrected ephemeris is Marsh's (1999) shifted by 0.25 of an orbit so the modulation of the central spike corresponded to the motion of the compact object. We present two orbital cycles for each line. In all lines we see the sinusoidal component that suggested the binary nature of GP Com. It zigzags between the two wings of the lines. The central spike appears in the trails as the vertical line fixed at low velocities. Doppler maps of the lines obtained using the maximum entropy method (MEM) are presented in the middle panels of Figs. 5 and 6. The central 


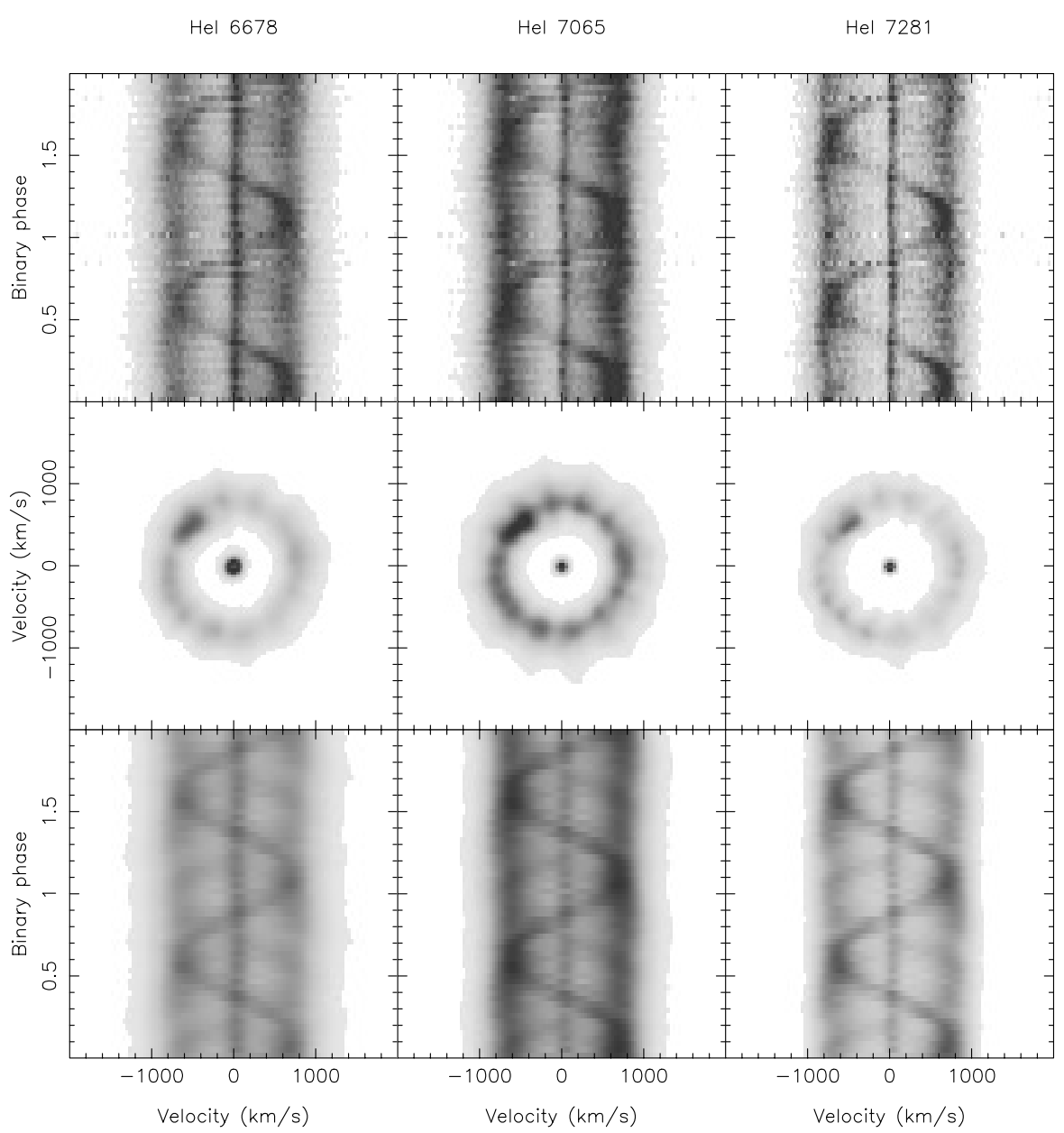

Fig. 6. Same as for Fig. 5 but for the three He I lines observed in the red. Notice that the intensity variations seen along the accretion disc on the three lines and more significantly in $\mathrm{He}$ I $\lambda 7065 \AA$ are the result of poor orbital phase coverage.

spike maps into the low velocity emission in the centre of the maps. The double wings located at $\sim 800 \mathrm{~km} \mathrm{~s}^{-1}$ either side of the rest wavelength map into an accretion disc around the central emission. Lastly, the "sinusoidal" component maps into emission coming from a region located on the accretion disc. This emission had previously been interpreted as coming from the bright spot (Marsh 1999). We notice that the bright spot shows a complex structure for some lines (i.e. He I $\lambda 6678 \AA$, He I $\lambda 7065 \AA$, He I $\lambda 7281 \AA$ ), stretched along the accretion disc in the maps. This behaviour, already seen by Marsh (1999), indicates that the bright spot is actually moving in a semisinusoidal fashion probably due to phase-dependent visibility in the emission regions, i.e. at some phases we see emission from the stream and at others emission from the disc near the stream. In the next section we present a variation of Doppler tomography that takes into account flux variability in the lines. By means of this new technique we see that the behaviour of the bright spot is rather unusual.

\subsubsection{Eccentricity of the accretion disc}

Another interesting effect seen in some of the lines (He I $\lambda 6678 \AA$, He I $\lambda 7065 \AA$, He I $\lambda 7281 \AA$ mainly) is that the accretion disc does not appear circular in the maps but somewhat elliptical. Once the motion of the white dwarf has been subtracted, a circular accretion disc would be seen in the trails of the lines as two vertical lines situated at the same distance at both sides of the rest wavelength. If the disc were elliptical, the vertical lines would not be at the same distance from the rest wavelength at all times. They would show higher radial velocities at orbital phases that correspond to a higher distance from the centre of the map. If we subtract the average spectrum from all spectra, we would be left with only the deviations from the circular disc.

To confirm the disc's ellipticity we measured the eccentricity in different regions of the Doppler map. In Fig. 7 we present a Doppler tomogram for GP Com obtained after adding up the three emission lines in the red part of the spectrum, He I $\lambda 6678 \AA$, He I $\lambda 7065 \AA$ and He I $\lambda 7281 \AA$. Before we added the spectra for the three lines, the motion of the white dwarf was subtracted in each case using the solutions given in Table 3. We then fitted elliptical curves to the equal flux contours in two different zones of the map. The procedure is described in Unda-Sanzana et al. (in preparation) but is briefly outlined here. It consists of two stages. After masking undesired regions (e.g. bright spot) the first stage is the selection of points at a certain level of flux, automatically picking them in such a way that the selection lies in a closed and continuous contour 


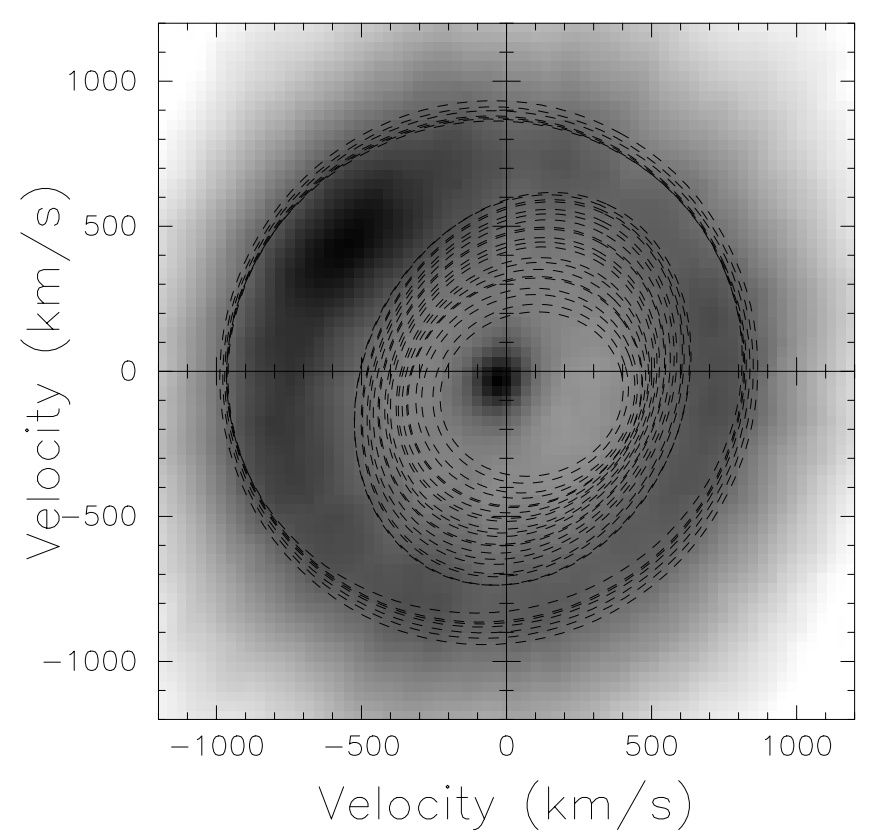

Fig. 7. Doppler tomogram obtained by adding up the signal from the three emission lines in the red spectrum: He I $\lambda 6678 \AA$, He I $\lambda 7065 \AA$, and $\mathrm{He} \mathrm{I} \lambda 7281 \AA$. Fits with ellipses to regions of equal flux were performed and plotted in the tomogram.

surrounding a point specified by the user (e.g. the origin). This is very straightforward for most "smooth" cases, but it is problematic for cases when large flux variations occur from pixel to pixel, typically near the tomogram's cusps or well into its outer regions. In those cases the selection would have to be done by hand by the user, so it is most reliable to exclude those regions from the process. The second stage is the use of the analytical ellipse-specific fitting algorithm by Fitzgibbon et al. (1999), in order to fit an ellipse to the selection. The outcome of the fitting is a number of parameters characterising the ellipse, which are later translated into physical quantities such as the rotation angle, size of semiaxes, etc. The selection is then bootstrapped and the fitting process repeated a large number of times in order to obtain uncertainties for the parameters. The first zone to which ellipses were fitted is the low velocity region in the map which corresponds to the outer regions of the accretion disc. The second zone is the high velocity region in the map which corresponds to the inner accretion disc. The top panel of Fig. 8 presents the eccentricity of the ellipses plotted in Fig. 7 versus semimajor axis. The central region of the accretion disc, where most of the emission is coming from, has not been fitted with ellipses because, as explained above, the first stage of the process is unreliable under the large flux variations of this zone. These large flux variations in the disc can be produced by the presence of the elongated bright spot or by the phase coverage of the data introducing artifacts.

The eccentricities of the ellipses fitted to the first zone give a consistent value of $\sim 0.6$ which indicates that the outer regions of the disc are not circularly symmetrical in flux. The bright spot was masked when we performed the fits so the asymmetry does not arise from its presence in the outer rim of the disc. For the second zone, the eccentricity near the boundary layer
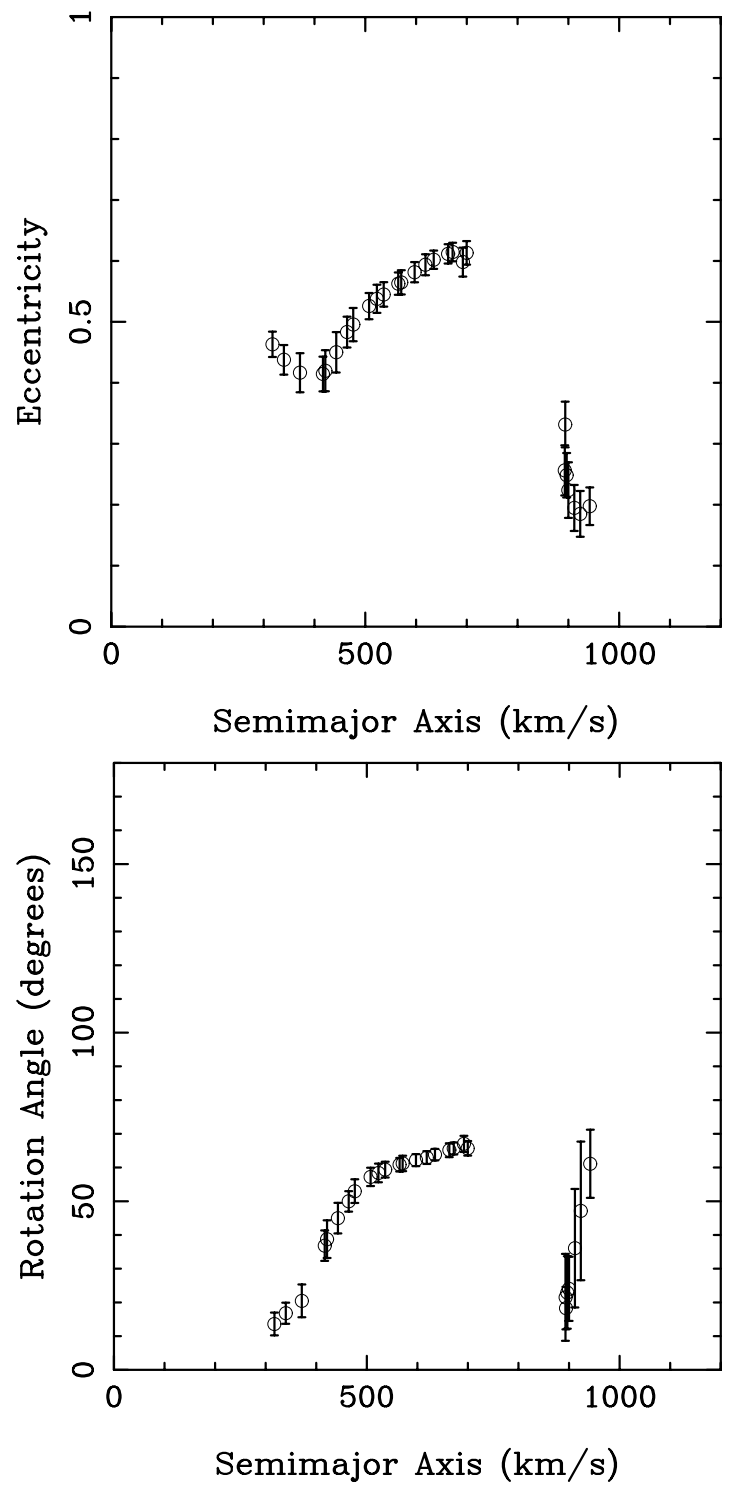

Fig. 8. The top panel represents the eccentricity of the ellipses fitted to the Doppler map in Fig. 7. As the ellipses get closer to the outer rim of the disc the eccentricity becomes constant with a value of $\sim 0.6$. The ellipses fitted to the inner regions of the disc start with a value for the eccentricity of 0.3 and then become more circular as they get closer to the white dwarf. The bottom panel represents the angle of the semimajor axis in the ellipse versus the length of the semimajor axis. The angle is measured from the positive $x$ velocity axis and increases anticlockwise. The angle increases as the ellipses get closer to the outer rim of the disc.

is $\sim 0.3$ and then it decreases to 0 soon after. This decrease is probably the result of getting closer to the white dwarf.

The bottom panel of Fig. 8 shows the angle of the semimajor axis for each ellipse. The angle is measured from the positive $x$ velocity axis and increases anticlockwise. We see that for the first zone the rotation angle increases for each ellipse as we get closer to the outer rim of the disc. As for the second region, the angle of the ellipses also increases as we get closer to the white dwarf.

According to Paczynski (1977), the maximum size for a very small pressure and viscosity accretion disc can be 
Table 5. Position of the bright spot and maximum radius of the largest stable orbit according to Paczynski (1977) (first value) and Henon (1969) for five different mass ratios. The position of the bright spot was calculated assuming that the angle between the donor star and the spot is between $58^{\circ}$ and $79^{\circ}$. Distances are given in terms of a, the distance between both stars in the system, and are measured from the compact object.

\begin{tabular}{ccc}
\hline \hline Mass ratio & bright spot (a) & Max. stable orbit (a) \\
\hline 0.01 & $0.474-0.387$ & $0.559 ; 0.472$ \\
0.02 & $0.452-0.345$ & $0.537 ; 0.466$ \\
0.03 & $0.443-0.325$ & $0.521 ; 0.459$ \\
0.04 & $0.438-0.312$ & $0.509 ; 0.454$ \\
0.05 & $0.436-0.304$ & $0.498 ; 0.449$ \\
\hline
\end{tabular}

approximated by the orbits of a test particle in the restricted 3-body problem. In the case of GP Com, where $q=M_{2} / M_{1}$ and $\mu=M_{2} /\left(M_{1}+M_{2}\right)$ have very close values, i.e. $\sim 0.02$ (Marsh 1999) we would expect the outermost stable orbit to be elliptical with eccentricity $e \sim 0.5$ (using the closer value for $\mu$ in Paczynski 1977). The eccentricity we measure in Fig. 8 is somewhat larger than this, $\sim 0.6$. If 3-body effects are the cause of the eccentricity seen in the disc, we would expect the semimajor axis of the elliptical disc to be perpendicular to the line that joins both components of the system in spatial coordinates. In velocity space this translates into the semimajor axis of the elliptical disc being parallel to the line that joins both components of the system, the white dwarf and the donor star. This argument implies that the donor star would be situated about $70^{\circ}$ anticlockwise of the positive $x$ axis in the velocity maps presented in Figs. 5-7, according to the rotation angle measured in the bottom panel of Fig. 8. Notice that we choose the closest orbit to the visible disc as the largest stable orbit. We do not find any significant emission other than that coming from the accretion disc at such position which could just be the result of the donor star being very dim. If the donor star were placed in that position in the Doppler maps, the angle between the positions of the donor star and the hot spot would be between $\sim 58^{\circ}$ and $\sim 79^{\circ}$. We have calculated the trajectory of the stream in velocity space for different values of the mass ratio $q$ and find that for the bright spot to be between $58^{\circ}$ and $79^{\circ}$ from the donor star, the size of the accretion disc would be as given in Table 5. In the table we also present the maximum radius of the largest stable orbit according to Paczynski (1977) and Henon (1969) which corresponds to the semi-major axis in an elliptical orbit. From the values in Table 5 we deduce that it could be possible that the eccentricity seen in the accretion disc would be due to 3-body effects as the values of the radii of the largest stable orbits and the positions of the bright spot are comparable for all the mass ratios considered (more so for values obtained according to Henon 1969). This argument implies that the ephemeris given in Sect. 3.4 might be incorrect and therefore the absolute orbital phases used throughout the paper are uncertain.

It is worth pointing out that Unda-Sanzana et al. (in preparation) have carried out a similar study for the $\mathrm{CV} U$ Gem and find that although the accretion disc is eccentric, the semi-major axis of the ellipses that fit the disc is not aligned with the line that joins the compact object and the donor star in the velocity maps. In U Gem the donor star is clearly seen in the spectra and therefore in the Doppler maps.

\subsubsection{Modulation Doppler maps}

We have mentioned in Sect. 3.4 that the bright spot emission shows deviations from a sinusoidal curve (see Figs. 5 and 6). These deviations are larger in the data presented by Marsh (1999) when GP Com seemed to be in a different state (as seen by comparing the average spectra). These deviations, probably caused by phase-dependent visibility, are directly linked with flux variability seen in the bright spot emission.

To study the flux variability in the emission lines as a function of orbital phase we use a new extension to Doppler tomography. The method is described in detail in Steeghs (2001, 2003), and splits the reconstructed Doppler maps in an image describing the constant contribution and two images describing the variable contribution. The first of these two maps represents line flux varying with the cosine of the phase, while the second represents flux varying with the sine of the phase. We are in particular interested in modelling the anisotropic emission from the bright spot region in GP Com. Since the strong central spike does contribute to the erratic flaring, but does not modulate smoothly in intensity with orbital phase, we masked out the low velocity regions of the line concerned. For all the lines, the achieved $\chi^{2}$ values were significantly better using the modulation mapping code, compared to the fits achieved by standard Doppler tomography. No significant residuals remained, indicating that most of the anisotropies present were indeed modulated on the orbital period and adequately prescribed by our modulation mapping code.

The persistent emission was discussed in Sect. 3.4 and revealed the accretion disc, the stream-impact region and the central spike to be the main emission line sources. Modulation maps show that the disc emission is only very weakly modulated, and that most of the time-dependent flux originates in the bright spot region. We thus limit our detailed discussion concerning the results from the modulation mapping to the properties of the bright spot. In Fig. 9, the modulation maps of the bright spot region are presented for He I $\lambda 5016 \AA$ and He I $\lambda 6678 \AA$. Considerable differences in the bright spot properties are found when comparing lines. All lines show a considerable ( $>5$ per cent) time-dependent emission component from the bright-spot region reaching for example an amplitude of 19 per cent in He I $26678 \AA$. The location and phasing of the variable bright-spot emission differs considerably from line to line but is generally positioned at smaller $V_{x}$ and $V_{y}$ velocities compared to its persistent counterpart for a given line. The lower $V_{y}$ velocities indicate that the modulated emission from the bright spot region has a smaller contribution from material moving with disc velocities, while the lower $V_{x}$ velocities indicate it is further out in terms of radius. Thus, to first order, the bright spot emission can be described by a steady component lying deeper into the disc and showing some mixing with disc velocities, with additional anisotropic emission localised 

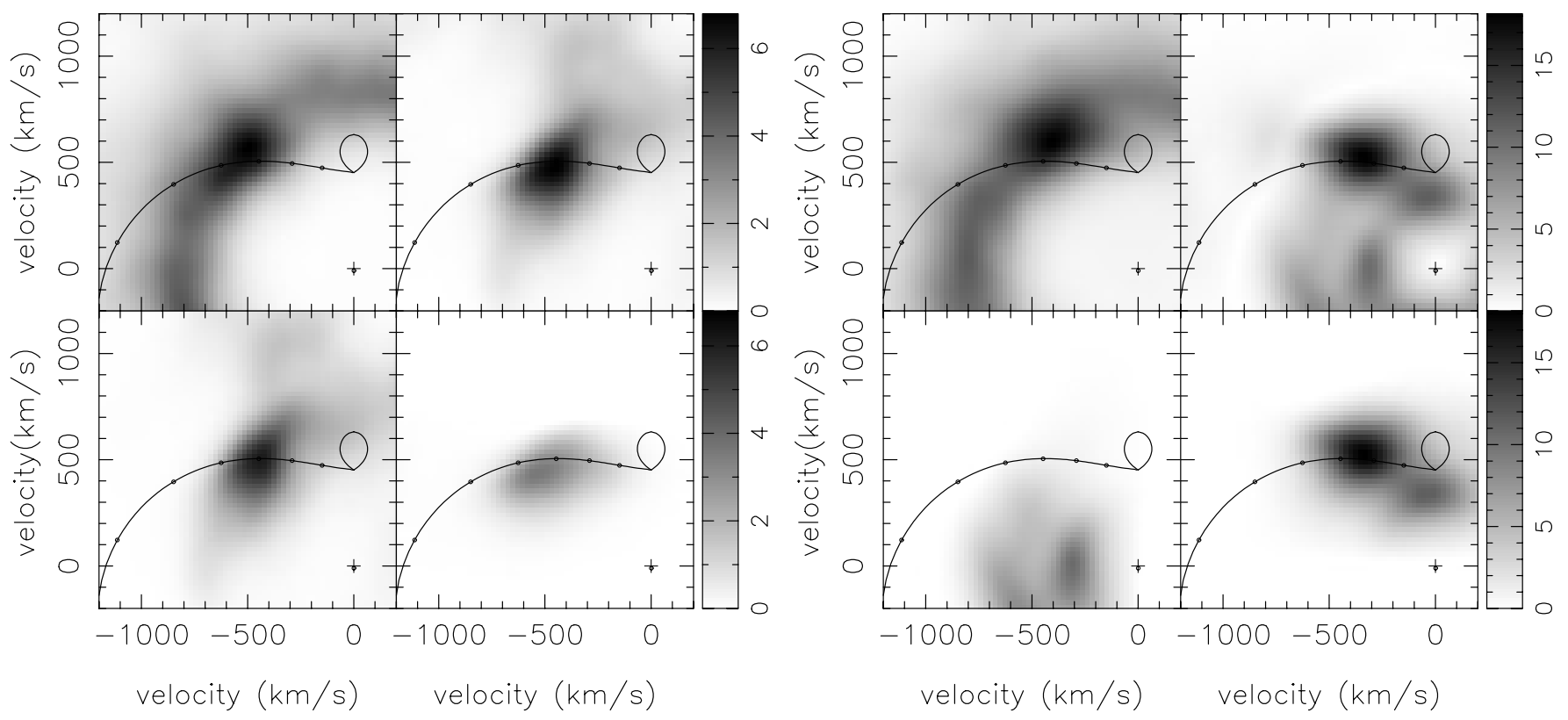

Fig. 9. Modulation Doppler images for He I $\lambda 5016 \AA$ (left) and He I $\lambda 6678 \AA$ (right). Only the parts of the maps close to the bright spot are displayed. The top left panels of both figures represent the constant part of the line emission, the top right the total amplitude of the modulated part of the emission. The grayscale wedge denotes the amplitude scale in percentage. The bottom two panels of both figures split the variable part in its cosine (left) and sine contributions. A $q=0.02$ ballistic stream trajectory is also plotted for reference.

along the incoming stream. In some lines, apart from this compact modulated spot, there is also extended modulated emission along the stream. In He I $\lambda 5016 \AA$, this stream emission modulates at 3 per cent and peaks around phase 0.15 , while in $\mathrm{He} \mathrm{I} \lambda 4472 \AA$ it dominates the variable emission and peaks close to phase 0.25 . In contrast, the He I $\lambda 4388 \AA$, He I $\lambda 6678 \AA$ and He I $\lambda 7281 \AA$ transitions do not show such an extended modulated component.

The modulation maps thus highlight the complex morphology of the bright spot emission in GP Com. Different lines are dominated by different parts of the disc-stream interaction region. Unfortunately, the uncertainty in both the mass ratio as well as the absolute phasing does not allow us to compare the observed velocities to a ballistic stream model in detail.

\section{UV spectra}

The UV spectra are equivalent to those presented by Marsh et al. (1995) but in this case the spectral resolution is higher and our spectra do not suffer from offsets in the acquisition. The combination of these two factors results in the blue wing of N V $\lambda 1239 \AA$ not overlapping with the geocoronal Lyman $\alpha$ emission (dashed section of the spectrum in Fig. 10), allowing us to see another NI line bluewards of it. The top panel of Fig. 10 shows an average of the 13 spectra with the principal emission lines labelled. Most of the lines are nitrogen, I, II, and $\mathrm{V}$ and possibly $\mathrm{C}$ I. Of most interest because of their strength are the NV lines, NV $\lambda 1239 \AA$ and N v $\lambda 1243 \AA$. These two lines seem to be composed of a central spike and broad wings like the emission lines in the optical (see Fig. 12 for an expanded view of these lines). Although we have labelled the feature on the left of N V $\lambda 1239 \AA$ as $\mathrm{N}_{\mathrm{I}}$, it

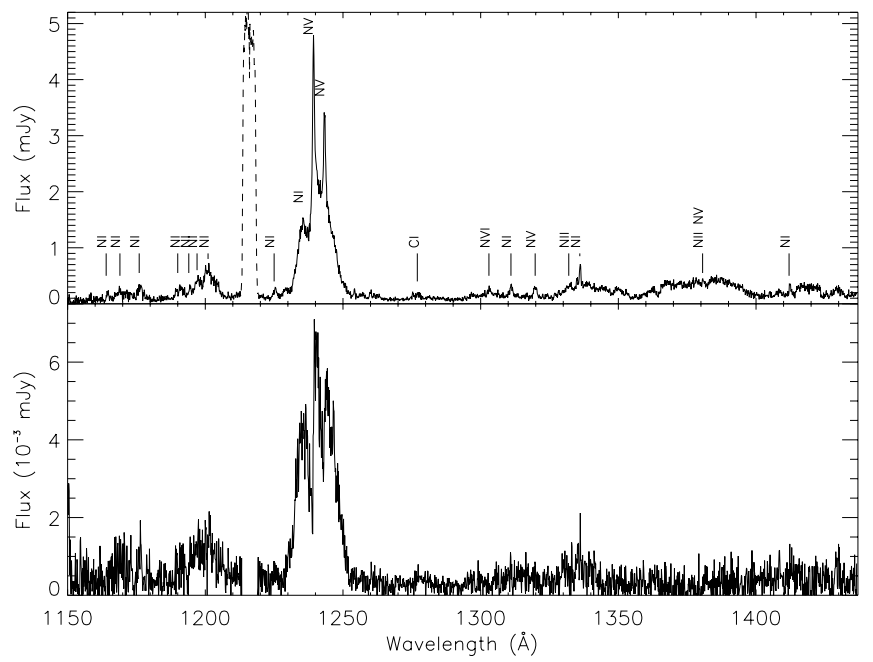

Fig. 10. Top panel: average HST spectrum of GP Com in the UV. Bottom panel: flare spectrum of GP Com in the same wavelength region. Most of the emission lines have been identified and labelled in the average spectrum.

is probably a combination of N I $\lambda 1235 \AA$ and the blue wing of N v $\lambda 1239 \AA$.

We computed Doppler maps (Marsh \& Horne 1988) of N V $\lambda 1239 \AA$ and N V $\lambda 1243 \AA$. They show the low velocity emission associated with the central spike in the lines and a ring-like structure situated at $\sim 1200 \mathrm{~km} \mathrm{~s}^{-1}$ in the case of $\mathrm{N} \mathrm{V} \lambda 1239 \AA$ and $\sim 1000 \mathrm{~km} \mathrm{~s}^{-1}$ in the case of N V $\lambda 1243 \AA$. These structures are associated with the accretion disc. The fact that the velocities associated to the disc are larger in the UV $\left(\sim 1000 \mathrm{~km} \mathrm{~s}^{-1}\right)$ than those seen for the optical emission lines $\left(\sim 800 \mathrm{~km} \mathrm{~s}^{-1}\right)$ is due to the $\mathrm{UV}$ emission being restricted to the 


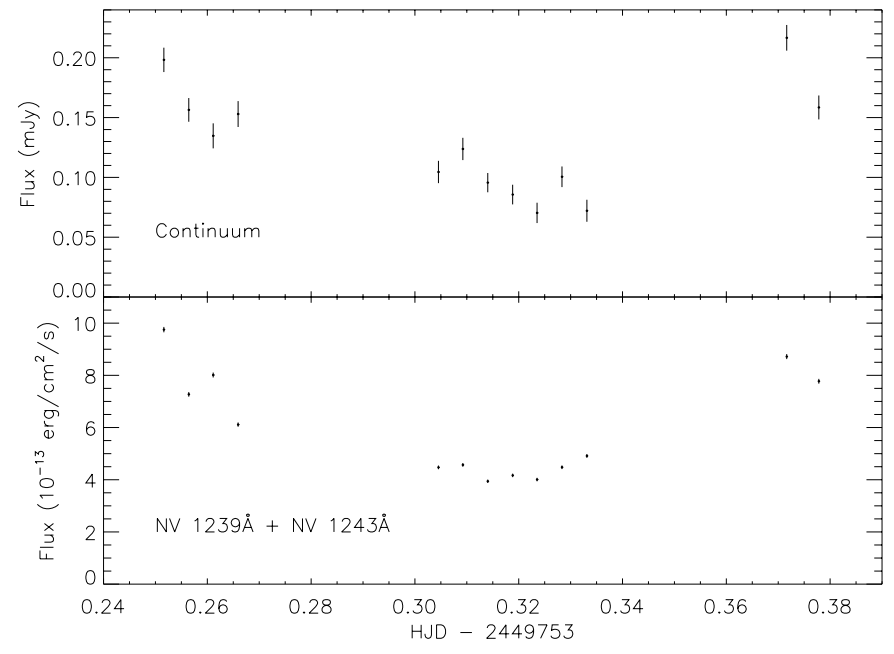

Fig. 11. Lightcurves of the UV continuum (top) and the sum of N V $\lambda 1239 \AA$ and N V $\lambda 1243 \AA$ (bottom).

inner regions of the disc. The low velocity emission also appears as a ring around the zero velocity reaching of the order of $100 \mathrm{~km} \mathrm{~s}^{-1}$. This is probably an artifact caused by the fact that we have assumed a zero systemic velocity when computing the maps when the real systemic velocity is not zero (see Marsh \& Horne 1988 for an explanation on the origin of artifacts seen in Doppler maps). These maps were obtained using only 13 spectra that, although they cover almost twice the orbital period, give very low resolution maps, full of artifacts in the form of radial features. This is why we do not display them. There is no clear presence of emission at the bright spot position in contrast with the data discussed by Marsh et al. (1995).

We notice that there are significant differences between each of the 13 spectra under study and conclude that there is a strong flare component superposed to the average spectrum. We present the lightcurves obtained for the continuum and the $\mathrm{N} \mathrm{V}$ lines in Fig. 11. Although the number of spectra obtained is quite small we can clearly see flux variations in the continuum and the emission lines. The data is divided into three subsets with gaps of 7 and 8 hours respectively between the subsets. The continuum and line flux variations follow the same trends, in contrast with the behaviour observed for the optical blue data. We observe flux varibility in two different timescales. Variations that lasts of the order of 20 min are seen during the two first subsets of data (it is unclear whether these are present during the third subset). We also see a difference in flux between the three subsets, the first and third appearing brighter than the second. In the same way discussed in Sect. 3.2 we calculate the flare component of the spectrum and display it in the bottom panels of Figs. 10 and 12. The two N V lines as well as the fainter N I lines contribute significantly to the flare spectrum. It is not clear whether the central spike is present in the flare spectrum. In contrast with Marsh et al. (1995) we do not detect any new features in the flare spectrum. We cannot confirm the presence of C IV $\lambda 1550 \AA$ as our spectrum does not cover that wavelength region. We do notice that there is no emission in the Si IV $\lambda 1400 \AA$ doublet region confirming that silicon is under-abundant in GP Com. If the presence of

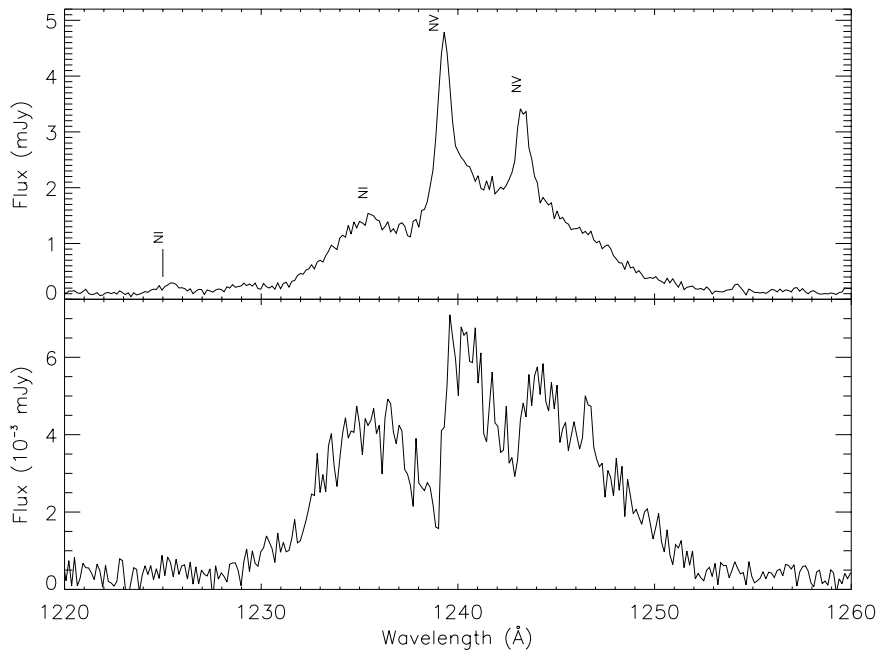

Fig. 12. Same as in Fig. 10 but expanded on the N v features.

nitrogen in GP Com is due to the action of the CNO process, the only explanation for the high abundance of nitrogen and low abundance of silicon in the system is that nitrogen-rich material was transferred from the progenitor of the accreting white dwarf to its companion during a common envelope phase.

\section{Conclusions}

We confirm the presence of the central spike in the flaring component of the spectra which indicates that its origin is probably the accreting star. We find that the central spike and the continuum vary in a similar ways which results in the central spike not being present for all lines in the flare spectrum obtained from the continuum normalised data. This is not the case for the broad component of the lines which indicates that they originate in different regions.

We detect the presence of a satellite peak bluewards of the central spike for three of the emission lines (i.e. He I $\lambda 4388 \AA$, He I $\lambda 4472 \AA$ and He I $\lambda 4922 \AA$ ) and suggest that they are neutral helium forbidden transitions excited by the presence of charged particles in the gas (the so called Stark effect). If these satellite peaks are indeed the result of Stark effect, we predict the presence of other lines associated with more neutral helium forbidden transitions at 3705, 4009, 3820, 4144 and specially $4026 \AA$ and encourage astronomers to observe GP Com as well as CE315 at those wavelengths. We believe this could be the key to understanding the origin of the until now elusive "central spike" and of the different spike redshifts seen for the different lines. The comparative study of GP Com and CE315 will also contribute to the understanding of these peculiar systems. We should keep in mind that although this explanation is very attractive, the predicted and measured separations between the forbidden and permitted components do not match so there might be other effects contributing to the behaviour of the central spike.

The Doppler maps computed from the spectra show the presence of an elongated bright spot and a slightly elliptical accretion disc. We measure an eccentricity for the disc of 0.6. 
Modulation Doppler maps show that the line emission from the bright spot is highly anisotropic. The anisotropic emission is formed at slightly larger radii, whereas the persistent emission shows some evidence of velocity mixing with the disc flow.

From the UV spectra we conclude that there is no sign of the presence of silicon in the system. The high nitrogen abundance combined with the low silicon abundance can be explained if nitrogen-rich gas was transferred from the progenitor of the white dwarf to the companion during a common envelope phase.

Acknowledgements. LMR was supported by a PPARC post-doctoral grant. DS acknowledges support from a PPARC Fellowship and is currently supported by a Smithsonian Astrophysical Observatory Clay Fellowship. EUS acknowledges the support of a PPARC Gemini, Fundación Andes studentship for Ph.D. studies at the University of Southampton. The William Herschel and the Jacobus Kapteyn telescopes are operated on the island of La Palma by the Isaac Newton Group in the Spanish Observatorio del Roque de los Muchachos of the Instituto de Astrofísica de Canarias. Based on observations made with the NASA/ESA Hubble Space Telescope, obtained at the Space Telescope Science Institute, wich is operated by the Association of Universities for Research in Astronomy, Inc., under NASA contract NAS 5-26555. These observations are associated with proposal \# 5689. The reduction and analysis of the optical data were carried out on the Southampton node of the STARLINK network.

\section{References}

Beauchamp, A., \& Wesemael, F. 1998, ApJ, 496, 395

Beauchamp, A., Wesemael, F., \& Bergeron, P. 1997, ApJS, 108, 559
Beauchamp, A., Wesemael, F., Bergeron, P., \& Liebert, J. 1995, ApJ, 441, L85

Cumming, A., Marcy, G. W., \& Butler, R. P. 1999, ApJ, 526, 890

Fitzgibbon, A., Pilu, M., \& Fischer, R. 1999, IEEE Trans. PAMI, 21, 476

Henon, M. 1969, A\&A, 1, 223

Lambert, D. L., \& Slovak, M. H. 1981, PASP, 93, 477

Marsh, T. R. 1989, PASP, 101, 1032

Marsh, T. R. 1999, MNRAS, 304, 443

Marsh, T. R., Horne, K., \& Rosen, S. 1991, MNRAS, 366, 535

Marsh, T. R., Wood, J. H., Horne, K., \& Lambert, D. 1995, MNRAS, 274,452

Nather, R., Robinson, E., \& Stover, R. 1981, ApJ, 244, 269

Nelemans, G., Portegies Zwart, S. F., Verbunt, F., \& Yungelson, L. R. 2001, A\&A, 368, 939

Osaki, Y. 1974, PASJ, 26, 429

Osawa, K. 1959, ApJ, 130, 159

Podsiadlows, Ph., Han, Z., \& Rappaport, S., in preparation [astro-ph/0109171]

Ruiz, M. T., Rojo, P. M., Garay, G., \& Maza, J. 2001, ApJ, 552, 679

Savonije, G. J., de Kool, M., \& van den Heuvel, E. P. J. 1986, A\&A, 155,51

Scargle, J. D. 1982, ApJ, 263, 835

Schneider, D. P., \& Young, P. J. 1980, ApJ, 238, 946

Steeghs, D. 2001, Proc. of the Astro-tomography workshop, Brussels, July 2000, ed. H. Boffin, D. Steeghs, \& J. Cuypers (SpringerVerlag), Lecture Notes in Phys. Ser., 573, 45

Steeghs, D. 2003, MNRAS, in press

Stone, R. P. S. 1977, AJ, 218, 767

Stover, R. 1983, PASP, 95, 18

Tsugawa, M., \& Osaki, Y. 1997, PASJ, 49, 75

Tutukov, A., \& Federova 1989, Sov. Astr., 33, 606

Tutukov, A., \& Yungelson, L. 1979, Acta Astron., 29, 665

Tutukov, A., \& Yungelson, L. 1996, MNRAS, 280, 1035

van Teeseling, A., \& Verbunt, F. 1994, A\&A, 292, 519 